\title{
ENERMONGRID: Intelligent Energy Monitoring, Visualization and Fraud Detection for Smart Grids*
}

\author{
MIGUEL LAGARES-LEMOS** \\ YULIANA PEREZ-GALLARDO*** \\ ANGEL LAGARES-LEMOS**** \\ JUAN MIGUEL GÓMEZ-BERBÍS*****
}

* This work is supported by the ITEA3 OPTIMUM project, ITEA3 SECUREGRID project and ITEA3 SCRATCH project, all of them funded by the Centro Tecnológico de Desarrollo Industrial -CDTI-.

** Master Biomedical Engineering. Nimbeo Estrategia e Innovación S.L., Madrid, España. E-mail: mlagares@nimbeo.com. ORCID: 0000-0003-1824-3740. Google Scholar: https://scholar.google.com/citations?view_op=list_works\&hl=es\&user=D6wo6ewAAAAJ.

*** PhD in Computer Science. Nimbeo Estrategia e Innovación S.L., Madrid, España. E-mail: yperez@nimbeo.com. ORCID: 0000-0002-2232-9582. Google Scholar: https://scholar.google.com/citations?user=1bF8dTwAAAAJ\&hl=es.

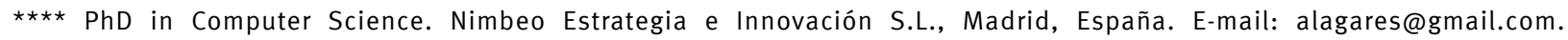
ORCID: 0000-0001-5310-301X. Google Scholar: https://scholar.google.com/citations?user=swVNmFoAAAAJ\&hl=es.

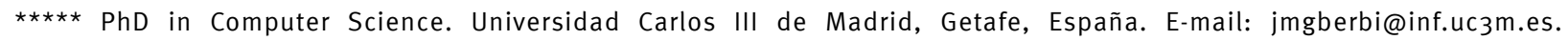
ORCID: 0000-0003-3902-9452. Google Scholar: https://scholar.google.com/citations?user=a2T86eoAAAAJ\&hl=es. 
COMO CITAR ESTE ARTÍCULO How to cite this article:

Lagares-Lemos, M. et al. (2020). ENERMONGRID: Intelligent Energy Monitoring, Visualization and Fraud Detection for Smart Grids. Revista Perspectiva Empresarial, 7(2-1), 44-55.

Recibido: 20 de agosto de 2020 Aceptado: 07 de diciembre de 2020
ABSTRACT The current obsolete electricity network is being transformed into net an advanced, digitalized and more efficient one known as Smart Grid. The deployment of an Automatic Metering Infrastructure will make an unseen quantity of rich information available in near real-time, processed to make decisions for the optimal energy production, generation, distribution, and consumption. This document presents an analysis of the ENERMONGRID tool, a tool used for intelligent energy monitoring, data visualization and fraud detection in electric networks.

KEYWORDS Energy, monitoring, fraud detection, smart grids, data visualization.

\section{ENERMONGRID: supervisión inteligente de la energía, visualización y detección de fraudes para las redes inteligentes}

RESUMEN La actual red eléctrica obsoleta se está transformando en una red avanzada, digitalizada y más eficiente conocida como Smart Grid. El despliegue de una infraestructura de medición automática permitirá disponer de una cantidad inédita de información abundante en tiempo casi real; procesada para tomar decisiones para la producción, generación, distribución y consumo óptimos de energía. Este documento presenta un análisis de la herramienta ENERMONGRID; una herramienta utilizada para la supervisión inteligente de la energía, la visualización de datos y la detección del fraude en las redes eléctricas.

PALABRAs CLAVE energía, supervisión, detección de fraude, redes inteligentes, visualización de datos. 


\section{ENERMONGRID: supervisão inteligente da energia, visualização e detecção de fraudes para as redes inteligentes}

RESUMO A atual rede elétrica obsoleta está se transformando numa rede avançada, digitalizada e mais eficiente conhecida como Smart Grid. A implantação de uma infraestrutura de medição automática permitirá dispor de uma quantidade inédita de informação abundante em tempo quase real; processada para tomar decisões para a produção, geração, distribuição e consumo ótimos de energia. Este documento apresenta uma análise da ferramenta ENERMONGRID; uma ferramenta utilizada para a supervisão inteligente da energia, a visualização de dados e a detecção de fraude nas redes elétricas.

PALAVRAS CHAVE energia, supervisão, detecção de fraude, redes inteligentes, visualização de dados. 


\section{Introduction}

The electrical grids of today managed by utility companies are complex systems with many different aspects that require expertise to operate successfully, such as grid management, data visualization, load prediction, loss prediction, and fraud prevention. The complexity of this domain is not only architectural, due to many different devices communicating and interoperating amongst the network, but there also exists a logical complexity, given the fact that incredible amounts of data must be properly managed in order to optimize the efficiency of the electric network.

Due to this difficulty and the many problems that can arise when dealing with energy load estimates, loss estimates, as well as fraud detection and prevention, the ENERMONGRID tool was developed to aid those entities in charge of managing an electric network, with the focus of doing so in Smart Grids.

This paper is made up of the following parts: (i) the introduction; (ii) a review of related works; (iii) the ENERMONGRID system is analyzed, detailing specific parts such as its architecture and involved elements, the restrictions and considerations that were taking into account when developing and testing the system, the anomalies that were found in the electric grid once the system was put into operation, the KPI's used to measure efficiency, as well as the amount of alerts that were fired during the duration of the analysis; (iv) finally, the conclusions.

\section{Related Work}

According to studies by the Galvin electricity initiative, in the United States the technologies Smart Grid will lower the costs of power supply and reduce the need for massive infrastructure investment in at least the next twenty years with a larger capacity electric grid. In the environmental aspect there is a great interest of the countries in developing policies and regulations that encourage the creation of social awareness with respect to consequences of greenhouse gases. The problem lies in the fuel used by traditional power generation plants and is produced during demand peaks that force the activation of special plants to be able to supply those additional energy requirements (García, Beltrán y Núñez, 2010). These plants are used only during these periods, with the resulting cost overruns - which have a direct impact on bills-. A very significant fact: in the United States, a developed country, $40 \%$ of carbon dioxide emissions come from electricity generation; while that only 20 $\%$ are caused by transport. This presents a huge challenge for the electricity industry in terms of climate change global.

There are currently many parallel activities related to standardization of Smart Grid networks. Since these activities are relevant to the same topic, it is some overlap and duplication of them is inevitable (Cleveland, 2008). There are several development agencies and standardization, among them:

(i) IEC Smart Grid Strategy Group: The International Electrotechnical Commission IEC - is the natural focal point for the electrical industry. It aims to provide a unique reference source for the many projects of Smart Grids that are being implemented around the world. It has developed a framework for standardization that includes protocols and reference standards for achieve interoperability of Smart Grid systems and devices (Díaz y Hernández, 2011).

(ii) National Institute of Standards and Technology - NIST-: It is not a body of standardization but has been designated by the government of the United States to manage the project of selecting a set of standards for the Smart Grid network in that country.

(iii) EU Commission Task Force for Smart Grids: Its mission is to assist the Commission and guidelines for European regulation and to coordinate the first steps towards the implementation of Smart Grid in the provision of the third energy package (Gordon and de Bucs, 2000).

(iv) IEEE P2030: It is an IEEE working group for the development of a guide for the interoperability of Smart Grid in the operation of energy technologies and information technology with the electrical power system -EPS— and the loads and end-user 
applications. Many demonstration projects are currently underway, and some results are available. The most representative initiatives in the field of Smart Grid are present in the United States, Europe, Japan and China.

\section{ENERMONGRID}

The ENERMONGRID tool is a tool used for intelligent energy monitoring, data visualization and fraud detection in electric networks. It is responsible for collecting data from the meters and transformation centers of the measurement system deployed in the metering infrastructure, also known as AMI, which stands for Advanced Metering Infrastructure (van der Meijden, Veringa and Rabou, 2010). This information is processed through the MDM module (Meter Data Management) that collects, consolidates, and manages this information.
The MDM module, among other tasks, also provides security through the anonymization of data, as well as advanced security measures; offers information to external systems through the communications module, through WEB REST services; manages the topological, cartographic and electrical information of the network; preprocesses and filters data relevant to the system.

The ENERMONGRID tool, via the use of algorithms developed by the different project members, calculates estimates, energy losses in the network, predictions, as well as energy balances. These results are treated as new reports, which have been designed for the project following the STG standard.

\section{Architecture}

The system architecture can be described with the following figure:

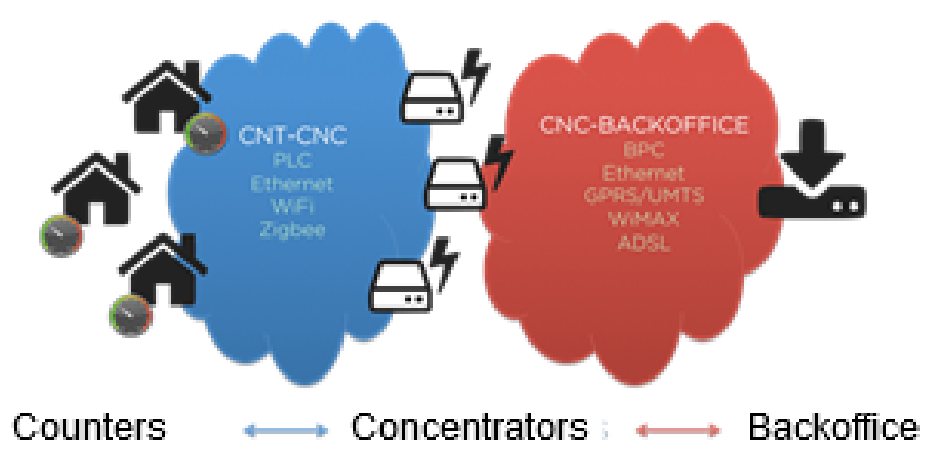

Figure 1. System Architecture. Source: author own elaboration.

The information flow of the architecture is given by three main elements: the meters, the transformation centers, and the BackOffice. The role of each one of these elements will be briefly described.

\section{Meters}

A meter is the device typically used to gather information regarding the energy consumption inside of a household or other location that consumes electrical energy. Most meters are analogical, but companies and technological drive have made it possible for these meters to be electrical devices in and of themselves, coining the term "Smart Meter." Smart Meters are especially important in the context and domain of Smart Grids and Smart Cities.

The role of the meter is to accrue the consumption rate of a location every fixed amount of time. Smart Meters can perform this data gathering duty over non-traditional methods such as OTA (over the air) by establishing a wireless 
connection with smart appliances. If this is not the case, however, a more standard approach is followed, which is simply to take measures at the power lines feeding the household or location. Once the meter has the information regarding energy consumption, it sends this information to a Transformation Center. This communication can be achieved via several different communication protocols, such as PLC, Ethernet, WiFi, or Zigbee.

(i) Transformation Center: The transformation center- $\mathrm{TC}$ - is the element in charge of aggregating the data obtained from a group of meters and then relaying it downwards towards the back office.

A single transformation center obtains information from many meters, so the relationship be a $1-\mathrm{N}$ relationship.

The TC can be seen as the intermediate step tasked with grouping lots of small measurements taken at meters and sent over low-throughput communication protocols, to sending great amounts of data through higher-throughput communication protocols and allowing the data to reach its ultimate destination.

The communication protocols used to relay the data from the transformation center to the BackOffice are typically BPC, Ethernet, GPRS/UMTS, WiMAX, or ADSL (Mowbray, 2013).

The ENERMONGRID tool allows to visualize the energy flow between transformation centers as can be detailed in the following figure:

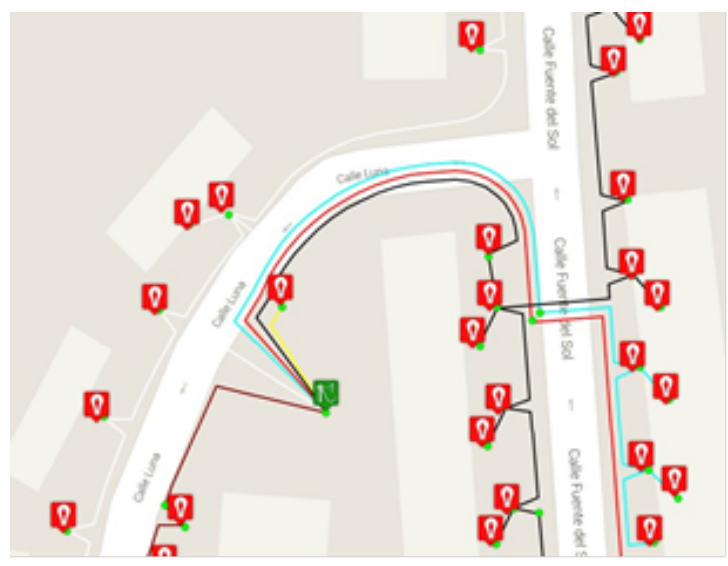

Figure 2. Energy flow of transformation centers. Source: author own elaboration. (ii) BackOffice: In this system architecture, the BackOffice be the destination of the data gathered from the meters. Once the data flows from the meters to the transformation centers, aggregated, and finally from the transformation centers to the BackOffice, the utility company is free to process the consumption data from each meter.

\section{Types of data and reports}

For ENERMONGRID to work the way it does and allow data prediction, visualization, and fraud prevention, it must first have access to the original reports obtained from electric utility services. A flow chart of the different types of reports is presented in the following figure, and will be explained in detail:

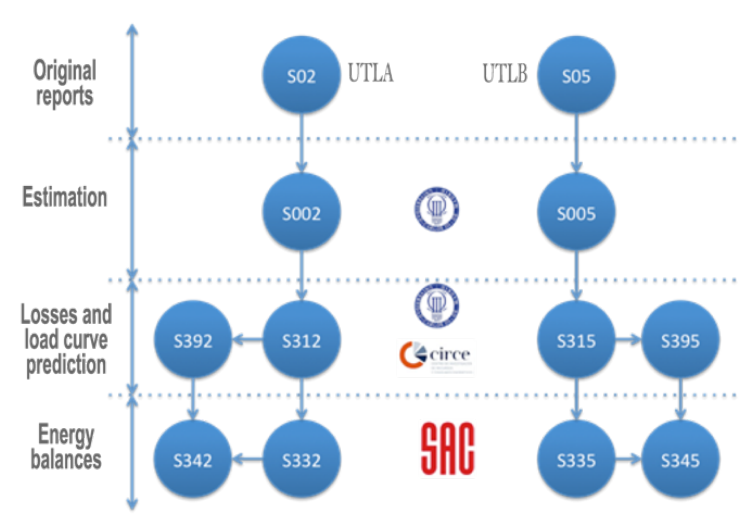

Figure 3. Types of reports and data dependencies. Source: author own elaboration.

In the first level of the data tree, we have the original reports. These are reports provided by the utility companies. These reports contain hourly data with information regarding the consumption observed by a specific meter. Each company has its own way of reporting this information, so two different formats are considered: the S02 file and S05 file.

On the next level, we have the estimation layer. This layer takes as input the original reports provided by the utility companies and processes the information in a way that allows to create estimates regarding further values. This process allows for a preliminary view at how data might change in upcoming moments. 
On the third level, we have the process in charge of predicting the curves of load on the system as well as potential losses. This sets the threshold for sampled values, allowing the detection of possible fraud-like activities in the network.

Finally, the last process involves the calculation of energy balances by using the previous layer's process output as a starting point.

It should also be noted that the tool internally processes information of real data (not estimated, nor of prediction), through its algorithms to have measurements of real energy balances, which can be compared with the estimates and predictions, as well as algorithms for the generation of alerts when certain conditions are met.

Through its web interface the tool allows access to all the information handled in it, both the monitoring and control of the network, the status of the various reports, the information of each report, cartographic information, consumption, voltages and intensities in nodes and stretches, power flows in sections, energy balances, alerts generated by the system, etc.

In the following figure, we can observe an interface which analyzes different metrics and KPI's of various types of reports detailed above. In this case, the S02,S352,S332 and S362 files are analyzed:

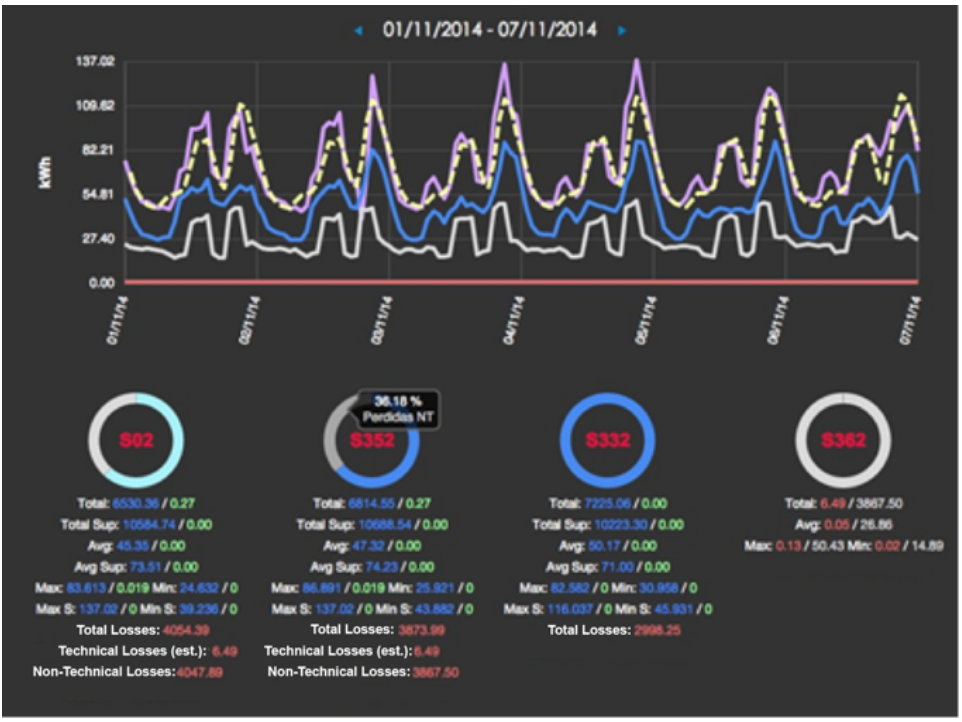

Figure 4. Analysis of energy balances. Source: author own elaboration.

\section{Considerations and restrictions}

In this section, some considerations and restrictions that have been present during the energy analysis process using the ENERMONGRID tool will be detailed.

(i) Samples with a quality bit other than '00' have not been considered: Samples with a quality bit of ' 00 ' have been, however, considered to calculate the Reading rate.
This modification has a side effect, because in certain scenarios when many of the samples of the meters come without the quality bit at ' 00 ' at a certain moment, when not taken into account, the total sum can produce very low values, throwing losses greater than those that actually exist in the network and which would be obtained by the calculations when the quality bit is taken into account. An example of this can be seen in the following figure: 


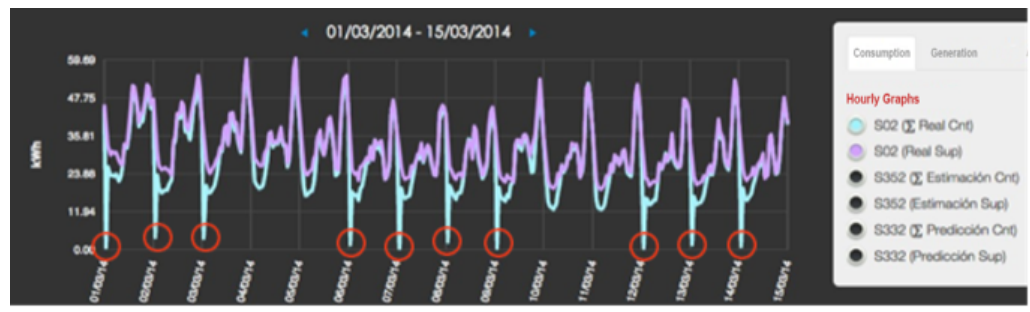

Figure 5. Side effect of discarding measurements with quality bit other than 'oo'. Source: author own elaboration.

The opposite situation can also occur, as in the following example in which one of the supervisors of one of the TC6 trades having values of Bc other than ' 00 ' is not taken into account, giving lower values of the sum of the supervisors compared to the sum of the meters. Resulting in negative losses, or in other words, apparent profits:

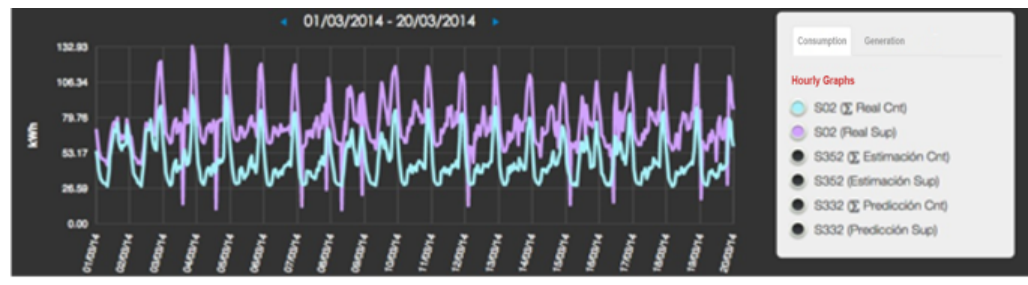

Figure 6. Apparent profits. Source: author own elaboration.

(ii) Meters that did not have a registered location in the system have not been considered in the analysis: The values obtained from meters not associated to a location were only used to adjust the reading rate value. This change arises because the estimator and the loss calculation algorithm do not take them into account and therefore, in order to compare with the real values, they have also been discarded inside the algorithms.

Given this condition, it can be observed that the consumption of the sum of meters is lower and therefore apparently increases the differences with the value of the supervisor, therefore yielding higher values of total losses.

\section{Real-time data anomalies}

In the following section, the real-time data anomalies that were observed during the analysis period of the ENERMONGRID tool will be detailed. (i) Supervisor values set to 0: There are certain scenarios (11.99\% of cases in the UTLA network and $1.58 \%$ for UTLB for the entire project) in which the supervisor shows consumptions equal to 0 , when there is consumption in the meters associated with them. All these values are associated with quality bits other than ' 00 ' for UTLB, but not for UTLA, where in some cases, not in the majority, the quality bit offers values to ' 00 '.

If we do this TC analysis for the UTLA network, in TC3 and TC4, whenever the supervisor gives a null value, it has the quality bit marked other than ' 00 '. It is not the same case for TC 1 and 2. It should also be noted that this is a case that has not been presented since April 2, 2014.

(ii) Abnormally high values: In some cases, abnormally high values have been detected in some meters that caused the balance to provide erroneous calculations and that did not allow to render in detail the data visualization graphs containing the balances of the TC. 
Twenty-nine abnormally high values have been detected over the entire duration of the project for the UTLA network.

(iii) Negative losses at specific moments: There are certain times when the total losses are negative, that is, the subtraction of the consumption data recorded by the supervisor minus the sum of the consumption record of all the meters associated with said supervisor (or TC) is less than 0.

The following figures show the number of moments (schedules, that they have been calculated through S02) where this circumstance occurs for the TC of the UTLA and UTLB network:

\begin{tabular}{lrrrr}
\hline & CT1 & CT2 & CT3 & CT4 \\
\hline Negative losses moments & 3587 & 3182 & 33 & 2657 \\
Moments negative losses without counting values Bcl= & 3588 & 3085 & 31 & 1722 \\
'00' Moments negative losses with all the perfect data & 0 & 2988 & 0 & 953 \\
\hline
\end{tabular}

Figure 7. Data from TC1-TC4. Source: author own elaboration.

\begin{tabular}{lcccc}
\hline & CT5 & CT6 & CT7 \\
\hline Negative losses moments & 158 & 11575 & 433 \\
Moments negative losses without counting values Bc!='00' & 158 & 11564 & 425 \\
Moments negative losses with all the perfect data & 0 & 10024 & 105 \\
\hline
\end{tabular}

Figure 8. Data from $\mathrm{TC}_{5}-\mathrm{TC}_{7}$. Source: author own elaboration.

The firstrow represents the totality of moments where total negative losses are recorded, which considering the number of records taken represent $0.20 \%$ of cases for UTLA and $0.18 \%$ for UTLB.

The second row shows the moments with negative losses when values with a quality bit other than ' 00 ' are not taken into account. Its value is slightly lower, which does not seem to have a relevant effect and simply reduces the number of moments due to the fact that these data are not taken into account in the sum.

The last row represents those moments with negative losses when all the data coming from the network have the quality bit at ' 00 '. This anomaly is still occurring (except in TC5), which should be studied further to determine the cause (meters or supervisors that measure incorrectly, meters that do not belong to this $\mathrm{TC}$ and are transmitting values to it).

\section{Reading rates of original reports}

In this section, the different reading rates obtained from analyzing reports across the different layers are detailed.

(i) UTLA: The reading rates obtained for the hourly reports (S02) are shown in the following figure for the different TC during the month of September:

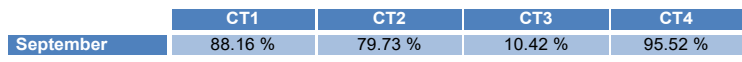

Figure 9. Reading rates of September. Source: author own elaboration.

As can be observed, the reading rate is around 80 $\%$ in September. The lowest rate can be found in TC3 given that it experienced technical communication problems during these dates, and the maximum values belong to TC4, which coincides with the TC that does not have any meter of industrial or commercial type (type 3 or 4), although this should not have a direct relationship, because these are not taken into account to establish the reading rate. If we do not consider the TC3 due to breakdown, the total rate is around $89 \%$.

Considering that TC3 has suffered a breakdown, this is what the ENERMONGRID tool provides in terms of data visualization for a fully operational TC, namely TC1, and the faulty TC3:

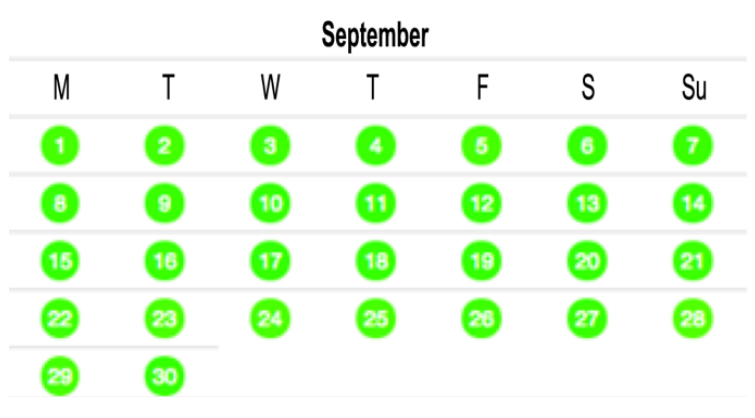

Figure 10. Reading rate of $\mathrm{TC}_{1}$. Source: author own elaboration. 


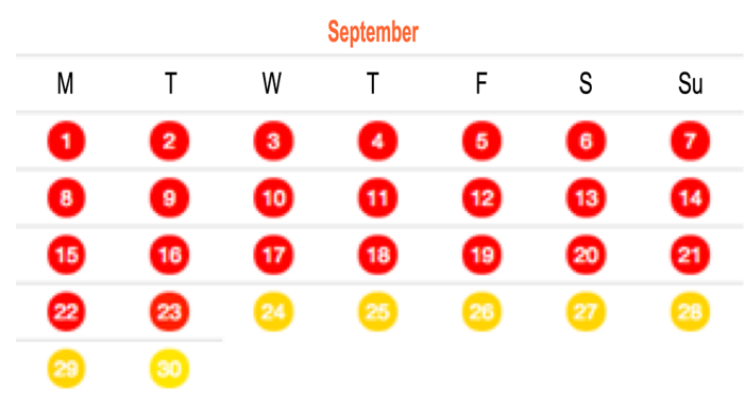

Figure 11. Reading rate of $\mathrm{TC}_{3}$. Source: author own elaboration.

(ii) UTLB: The reading rates obtained for the hourly reports (S02) are shown in the following figure for the different TC during the months of August and September:

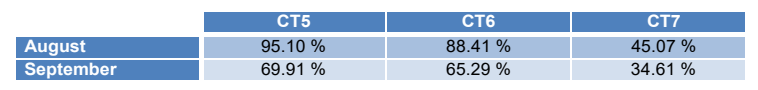

Figure 12. UTLB Readings. Source: author own elaboration.

As we can observe, the reading rate is around $47 \%$ in September, while in August it was $63 \%$.
Analyzing the availability of reports in September (next figure), we see that the reason for the lowest percentage during the month of September, is due to the lack of availability of reports on certain days.

\begin{tabular}{|c|c|c|c|c|c|c|c|c|c|c|c|c|c|}
\hline \multicolumn{7}{|c|}{ August } & \multicolumn{7}{|c|}{ September } \\
\hline M & $T$ & W & $T$ & $F$ & S & SU & M & $T$ & W & $T$ & $F$ & $S$ & SU \\
\hline & & & & (1) & 2 & (3) & (1) & 2 & (3) & (4) & (5) & (6) & ( \\
\hline$(4$ & (6) & (6) & (7) & 0 & 0 & (1) & 0 & 0 & (1) & (11) & (12) & (1) & (1) \\
\hline (11) & (12) & (1) & (1) & (1) & (1) & (1) & (1) & (1) & (1) & (1) & (1) & (2) & (2) \\
\hline (1) & (1) & (2) & (21) & (2) & (2) & (24) & (2) & (2) & (2) & (2) & (2) & (2) & 궁 \\
\hline (2) & (2) & (2) & (2) & 20 & (2) & (3) & (2) & (2) & & & & & \\
\hline
\end{tabular}

Figure 13. Availability of reports. Source: author own elaboration.

\section{KPIs of Energy Balances}

(i) UTLA: The UTLA values corresponding to the energy balances for September for UTLA and for August 2014 for UTLB are shown below (since the reading rate is better in that month). The KPIs are based on the real hour data (S02), except for the case of technical losses that are obtained from the estimated ones. These data can be consulted through the ENERMONGRID tool.

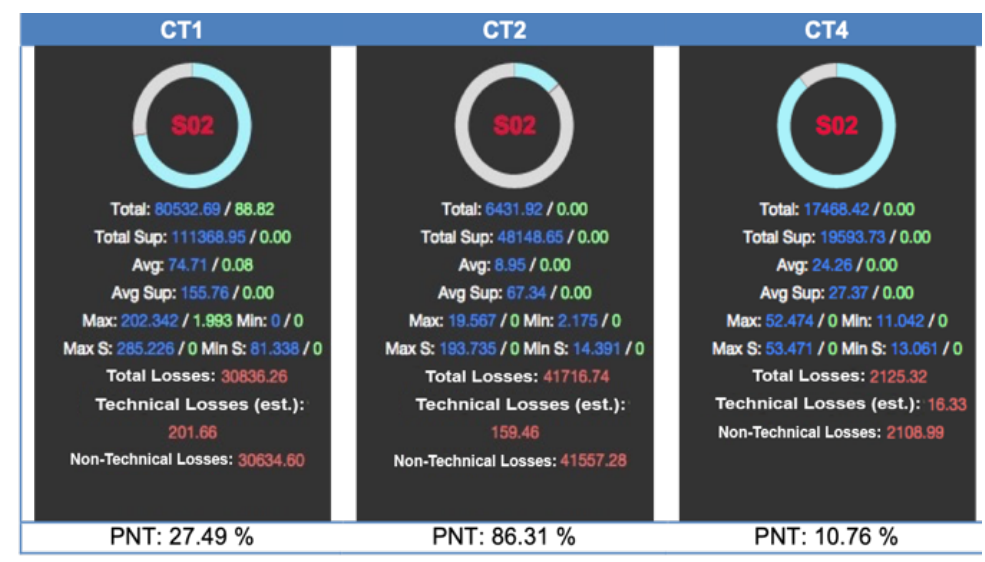

Figure 14. UTLA analysis. Source: author own elaboration.

As can be observed the non-technical losses are high, especially in TC1 and TC2, which have type 3 and type 4 meters, which, being not telemanaged and registered in the system, virtually increase non-technical losses. This can be seen in the following figure, which shows the supervisor's graph (in purple) and the sum of the meters (blue), in addition to the technical losses (red). The graph of the supervisor is far above the consumption reported by the meters. 
(ii) UTLB: The UTLB values corresponding to the energy balances for September for UTLA and for August 2014 for UTLB are shown below (since the reading rate is better in that month). The KPIs are based on the real hour data (S02), except for the case of technical losses that are obtained from the estimated ones. These data can be consulted through the ENERMONGRID tool.

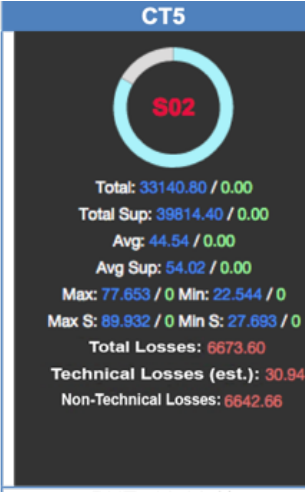

PNT: $16.68 \%$
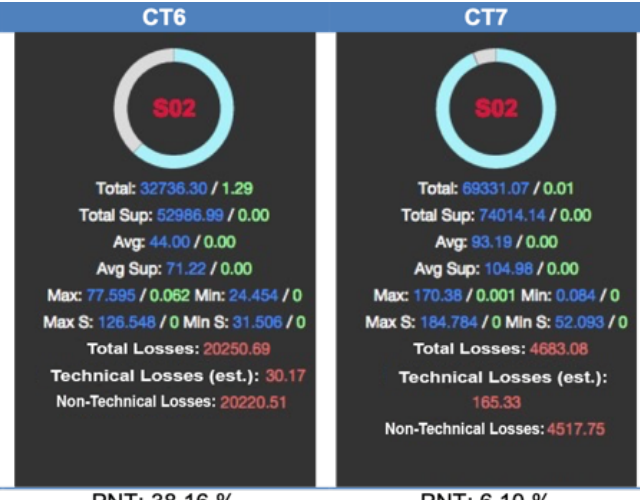

PNT: $38.16 \%$

PNT: $6.10 \%$

Figure 15. UTLB analysis. Source: author own elaboration.

As can be observed the non-technical losses are more moderate than in the UTLA network, since there is no existence of type 3 or 4 meters. The TC7 offers the best performance values and the TC6 registers apparently high values for this type of TC. The following figures show these two cases, where you can see the supervisor's graph (in purple), the sum of the meters (blue), and the technical (red) and non-technical (white) losses.

It is relevant that the TC7 in which the lowest reading rates were presented, offers the best results. This confirms that the meters that the system considers are probably meters written off or that are not yet operating.

\section{Alerts}

In the following section, details regarding the alerts set off $b$ the ENERMONGRID will be detailed, namely, the UTLA and UTLB alert values will be analyzed.

(i) UTLA: The system controls the tensions in the nodes and the saturation in the sections to generate alerts when the marked thresholds are passed: $7 \%$ deviation in voltage at nodes in the $230 \mathrm{~V}$ reference voltage; $90 \%$ saturation limit in sections.
Since September 2014, 3917 alerts have been registered, 13 regarding the saturation in sections and the rest due to deviations.

(ii) UTLB: The system controls the tensions in the nodes and the saturation in sections to generate alerts when the marked thresholds are passed: $7 \%$ deviation in voltage at nodes in the $230 \mathrm{~V}$ reference voltage; $90 \%$ saturation limit in sections.

Since November 22, 2014, 737 alerts have been registered, all due to the tension in nodes.

\section{Work approach}

The project will be validated with several procedural challenges that we will detail below:

(i) Testing with various stakeholders to see how the system is affected by various people or businesses it is understood that not all participants need to understand and address the needs and functionality of the system. 
(ii) Checking the complexity of the system, in addition to checking the good performance of the system. Some aspects will be sensitive to human response and interaction, while others will require instant and automated responses.

(iii) The security of the cybernetic systems will be checked to validate that the system is safe. Information security technologies are not sufficient to achieve secure operations if policies, risk assessment and training are not applied.

(iv) The intelligent network is an evolving goal, so you have to study all the capabilities it has.

As discussed in this article, there are many areas of work, but especially for ICTs necessarily involved in this transition.

There are numerous factors to consider and steps that can be taken now to get ready. The interesting thing is that there is a lot to do and no country leads to the moment a very marked lead in the definitive development of a system like ENERMONGRID.

\section{Conclusions}

The ENERMONGRID management tool complies very closely with the objectives presented in the project. It is true that the tool does not manage the network, because it was not intended to operate on the network, since access was restricted. However, the functionality that has been provided in other cases exceeds the initial claims, because in addition to collecting, pre-processing and analyzing all information safely through the MDM module, it also serves as a tool to establish communications with external systems and their visual function has been seen working on many other aspects not considered at first.

As future work we will seek to increase the completeness of ENERMONGRID to help more entities in charge of managing an electricity network, with the focus on creating a large smart grid. We will analyze the ENERMONGRID system, considering the elements involved, the restrictions that were taken into account when developing and testing the system, solving the anomalies that it presented and being able to develop a competitive system that can be used by any entity.

\section{References}

Cleveland, F. (2008). Cyber security issues for Advanced Metering Infrasttructure (AMI). In IEEE Power and Energy Society General Meeting - Conversion and Delivery of Electrical Energy in the $21^{\text {st }}$ Century, Pittsburgh, USA.

Díaz, C.A. y Hernández, J.C. (2011). Smart Grid: las TICs y la modernización de las redes de energía eléctrica - Estado del Arte. Sistemas \& Telemática, 9(18), 53-81.

García, A., Beltrán, P. y Núñez, S. (2010). Una aproximación al concepto de frontera virtual. Identidades y espacios de comunicación. Revista Latina de Comunicación Social, 65, 16.

Gordon, J.J. and de Bucs, F.H-S. (2000). A Model of Self-Similar Data Traffic Applied to Ethernet Traces. In Kouvatsos, D. (Ed.), Performance Analysis of ATM Networks (pp. 3-21). Boston, USA: Springer.

Mowbray, T.J. (2013). Cibersecurity: Managing Systems, Conducting Testing, and Investigating Instructions. New York, USA: Wiley.

van der Meijden, C., Veringa, H. and Rabou, P. (2010). The production of synthetic natural gas (SNG): A comparison of three wood gasification systems for energy balance and overall efficiency. Biomass and Bioenergy, 34(3), 302-311. 